\title{
Letter to the Editor
}

\section{Rigid ventilating video bronchoscope with forceps for bronchoscopic foreign body removal in children}

Sir,

Bronchoscopic foreign body removal is a potentially dangerous and challenging procedure in pediatric surgery. ${ }^{1}$ bronchoscopy under general anaesthesia is the gold standard of diagnosis and management of foreign body aspiration. ${ }^{2}$ A large ventilating channel and better control over the tip of the instrument and cheaper instrument are the merits of rigid bronchoscopy over flexible one. Traditionally a rigid tube alone is used for this purpose which has extreme limitations of vision and it is risky. ${ }^{3}$ Foreign body aspiration typically occurs in 6 to 18 month age and the size of glottis is very small at this age. In Indian children who are small and malnourished the large assembly of sheath and telescope mounted forceps does not pass through the glottis. To overcome the limitations of the traditional equipment I designed my own bronchoscopy equipment by my 15 year of experience in bronchoscopy. This type of device is reported for the first time in medical literature and patent is filed for it at Mumbai office.

The aims and objectives were to develop an ideal instrument for bronchial foreign body removal. To eliminate problems in conventional instruments such as blurring of vision, poor vision, large size of instrument, long operative time and operative morbidity and high cost etc.

Last 12 months I performed 21 bronchoscopies for bronchial foreign body with this instrument in Jawale Institute of Pediatric surgery. Age range was 7 months to 31 months. Maximum follow up was 31 months and minimum of 6 months. Mortality was zero and morbidity was negligible.

The instrument is a 5 in 1 device and is a genius merger of a telescope, LED light source, Ventilating bronchoscope sheath and bronchoscopic foreign body removal forceps. It is described for the first time in the medical literature. A patent for the device is registered at Mumbai office. A video camera with 4 LEDs is fitted at the tip of a rigid tube made up of surgical steel and has USB output (Figure 1A). 10 wires bundled into a single core of one $\mathrm{mm}$ pass through the empty tube and connected to another end of the instrument to an analogue to USB converter (Figure 1A). The tube has holes on each side for ventilation. It has a ventilating port. A foreign body removing peanut forceps is welded at $6 \mathrm{O}$ ' clock position to the tube for removal of bronchial foreign bodies. The jaws of the forceps are curved and have a gap in between which avoid crushing of a soft foreign body like a peanut. A handle moves the forceps at the tip. There are no fiber optics and rod lenses, hence no image loss and has excellent image quality. (Figure 2B, C) Outer transverse diameter of the instrument is $4.5 \mathrm{~mm}$ and vertical diameter of $5.5 \mathrm{~mm}$. The working length is $50 \mathrm{~cm}$. Suction channel is not given to the instrument as it will increase its diameter. A USB cable connects the instrument to the computer desktop, laptop, android device or a mobile phone. A free software is given along the instrument with facility for display and recording. Sterilization is done by formalin chamber or ethylene oxide gas.

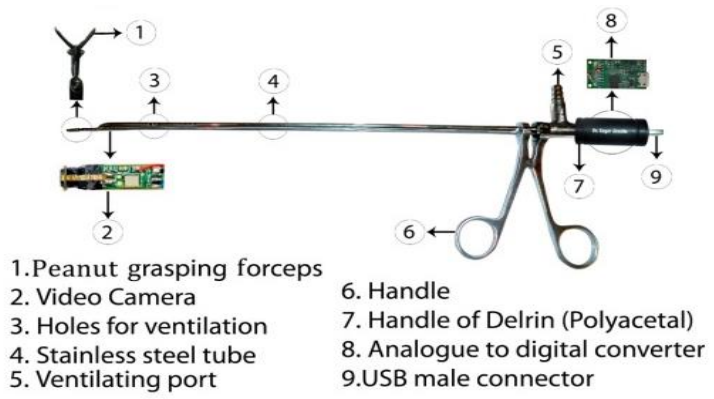

Figure:-1A

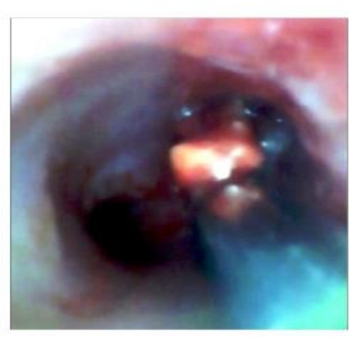

Figure:-1B

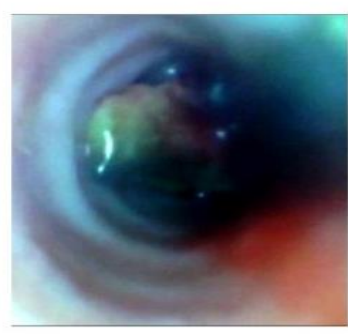

Figure:-1C
Figure 1: Rigid ventilating video bronchoscope.

The camera used has following Specification, typeAnalog Camera, Image Sensor: 1/18" CMOS, Size: Dia3.5 $\times$ L14mm, FOV: $54^{\circ}$, SNR: $\geq 42 \mathrm{~dB}$, Video format: NTSC, FPS: 60Fps, Effective Pixels: 750 (h) $\times 350(\mathrm{v})$, Power Supply: DC3.3V 3.6V, Depth of Field: $1-5 \mathrm{~cm}$, Fixed focus $1-5 \mathrm{~cm}$, Resolution: 320 by 240.

The procedure for bronchoscopy by the instrument is the same as described in medical literature. ${ }^{4-6}$ The foreign body grasped is shown in Figure 1B (Peanut) and Figure 1C (Mango pickle). 
The instrument was commercialized for a reasonable cost of USD 300. The instrument is easily worth USD 10,000 as a telescope (USD 3000), a LED light source (USD 1,500) endo camera (USD 4000) and ventilating bronchoscope (USD 1,500) are not necessary. It makes the instrument the cheapest commercially available instrument in the world.

The instrument is extremely light weight and weighs only 150 gm making it lightest bronchoscopic assembly compared to the conventional instrument with endo camera attached. Bronchoscopic foreign body removal takes only 15 mins with instrument compared to 60 mins for the conventional.

The instrument has best image quality (Figure $1 \mathrm{~B}$ and Figure 1C) as there is no image loss due to rod lens. The field of view is also excellent instead of the narrow tunnel vision of the conventional instrument. The ventilating ability and attached forceps makes reduces the risk and operative time dramatically. The instrument can record videos and still pictures very easily for documentation and for medicolegal purpose. The ventilating rigid video bronchoscope with forceps is safe, affordable, light weight and compact instrument. It is likely to revolutionize bronchoscopic foreign body removal procedure in India and other third world countries.

\section{Sagar Jawale ${ }^{1 *}$, Parthapratim Gupta ${ }^{2}$, Bharti Kulkarni ${ }^{3}$}

Department of Pediatric Surgery, ${ }^{1}$ Jawale Institute of Paediatric Surgery, Jalgoan, Maharashtra, ${ }^{2}$ Institute of Child Health, Kolkata, Wes Bengal, ${ }^{3}$ D.Y. Patil Medical College, Nerul, Maharashtra, India

\section{*Correspondence to}

Dr. Sagar Jawale, E-mail: drsagarjawale@gmail.com

\section{REFERENCES}

1. Hasdiraz L, Oguzkaya F, Bilgin M, Bicer C. Complications of bronchoscopy for foreign body removal: experience in 1035 cases. Annals of Saudi medicine. 2005;26(4):283-7.

2. Mehta D, Mehta C, Bansal S, Singla S, Tangri N. Flexible bronchoscopic removal of a three-piece foreign body from a child's bronchus. Australas Med J. 2012;5(4):227-30

3. Steve R, Thornington RE. Paediatric bronchoscopy. Continuing Education in Anaesthesia Critical Care \& Pain. 2005;5(2):41-4.

4. Farrell PT. Rigid Bronchoscopy for foreign body removal: anaesthesia and ventilation. Paediatr Anaesth. 2004;14(1):84-9.

5. Naragund AI, Mudhol RS, Harugop AS, Patil PH, Hajare PS, Metgudmath VV. Tracheo-Bronchial Foreign Body Aspiration in Children: A One Year Descriptive Study. Indian J Otolaryngol Head Neck Surg. 2014;66(Suppl 1):180-5.

6. Haddadi S, Marzban S, Nemati S, Ranjbar Kiakelayeh S, Parvizi A, Heidarzadeh A. Tracheobronchial Foreign-Bodies in Children; A 7 Year Retrospective Study. Iran J Otorhinolaryngol. 2015;27(82):377-85.

Cite this article as: Jawale S, Gupta P, Kulkarni B. Rigid ventilating video bronchoscope with forceps for bronchoscopic foreign body removal in children. Int $\mathbf{J}$ Otorhinolaryngol Head Neck Surg 2019;5:1129-30. 